\title{
Circuit Level Performance of FETS: A Comparative Study of Propagation Delay with Half Adder
}

\author{
Maskura Nafreen ${ }^{1}$, Nusrat Ara ${ }^{2}$, Md.Rakib Hasan ${ }^{3}$, MD.Imran Hossain ${ }^{4}$, Fariha \\ Rahman $^{5}$ \\ 1,2,3,4,5 (Department of Electrical and Electronic Engineering, Ahsanullah University of Science and Technology, \\ Bangladesh)
}

Abstract: This paper deals with the propagation delay comparison of half adder with different FETs; such as MOSFET, CNTFET, FINFET. Nanotechnology is the promising field which functions at the molecular level to replace the conventional use of classical CMOS. By simulation, the best part is got that CNTFET shows lesser delay for half adder circuit.

Keywords: CNTFET, MOSFET, FINFET, Hspice, Nanotechnology, propagation delay evaluation, CMOS, half adder, nanotube devices.

\section{Introduction}

Popularized word "Nanotechnology" is the process, in which the arrangement of individual atoms is properly controlled by manipulating structure, length within scale of one-billionth of a meter. [3]The Nano devices can be used for efficient purposes in microelectronics, power electronics, aerospace etc.[4] Carbon nanotube field-effect transistor (CNTFET) and Fin field-effect transistor (FINFET) are very popular nano scaled transistors nowadays.[2] Propagation delay expresses certain time which is required for a signal to move from input to output and it's a major factor for a device.[11]In this paper, the simulation results will show that CNTFET has better propagation delay character with the reasons working behind it.[10]

\section{Methods}

In this paper, timing diagrams of CMOS based half adder circuit is shown by using Synopsys Hspice software (version 2008.03) which is an open source analog electronic circuit simulator.[5]While using MOSFET based circuits, the cell library is already existed in default list. But for simulating CNTFET and FINFET based circuits, different cell libraries and net lists have to be generated. For CNTFET library, License agreement of Stanford University is used and for FINFET library BSIM-CMG models are used which are characterized as a standard library.[6][7] It is developed by using predictive technology (PTM).[8][9] From the net lists, measured values of different points of circuits are plotted using another software Matlab which provides a numerical environment allowing programming language of fourth-generation. It shows the difference among the transistors' performance in same graphs as characteristics curves. From relative results, it is seen that propagation delay analysis of CNTFET is comparatively better than other two.[1]

\section{Findings And Argument}

Half adder circuit is simulated using MOSFET, CNTFET and FINFET for different netlists to examine the switching delay among those.
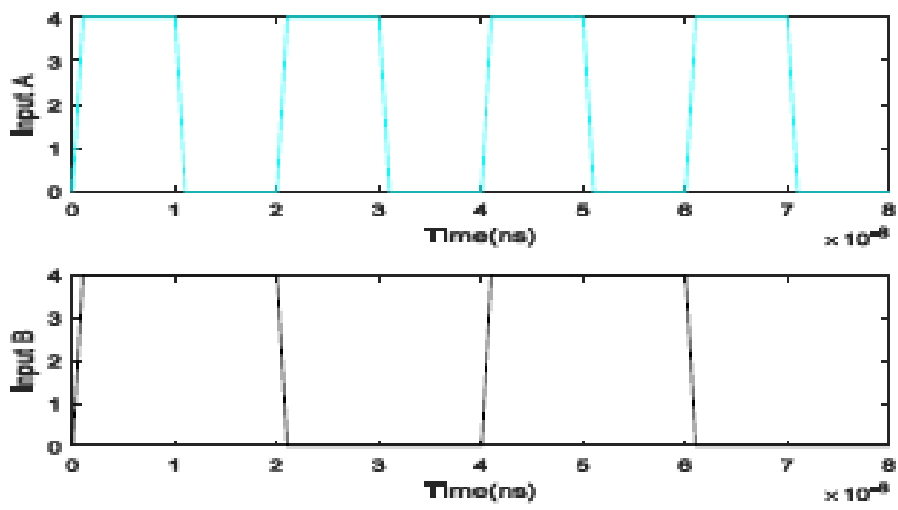

Fig. 1: The input pulses for half adder circuit 


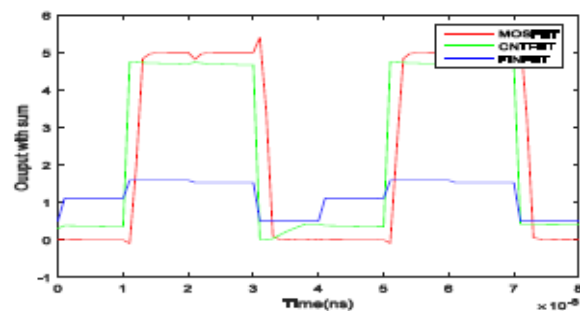

(a)

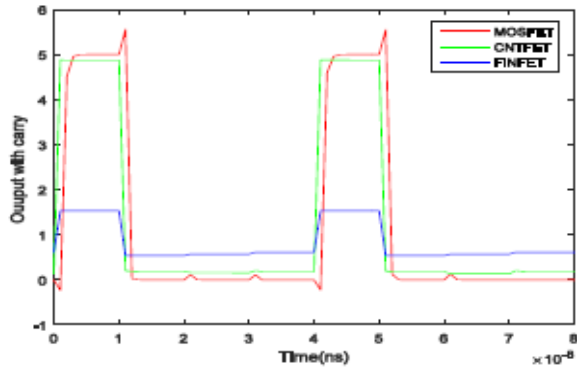

(b)

Fig. 2(a) Output diagram for a Sum bit of a half adder (b) Output diagram for a carry bit of a half adder

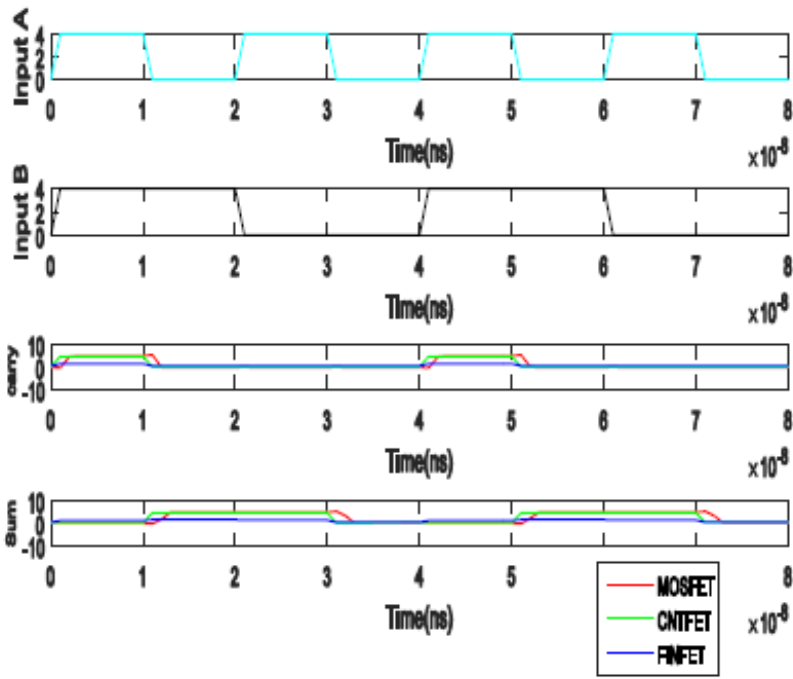

Fig. 3 Overall Timing diagram for a half Adder with different topology

In Fig. 1, Fig. 2 and Fig. 3, black and cyan colors are used to show two input signals. Red, green and blue respectively show timing diagram of MOSFET, CNTFET and FINFET. The propagation delay is symbolized by Tpd which is the summation of two parts-time delay of high to low (TpHL) and time delay of low to high (TpLH). It is measured from the difference of the instantaneous response value and the given input.

Table 1: Time delay analysis for three FETS in half adder circuit

\begin{tabular}{|c|c|c|c|}
\hline & MOSFET & CNTFET & FINFET \\
\hline For Sum & $\begin{array}{c}\mathrm{T} \mathrm{pLH}=0.033 \mathrm{~ms} \\
\mathrm{TpHL}=0.067 \mathrm{~ms} \\
\text { Total } \mathrm{Tpd}=0.1005 \mathrm{~ms}\end{array}$ & $\begin{array}{c}\mathrm{TpLH}=0 \mathrm{~ms} \\
\mathrm{TpHL}=0 \mathrm{~ms} \\
\text { Total } \mathrm{Tpd}=0 \mathrm{~ms}\end{array}$ & $\begin{array}{c}\mathrm{TpLH}=0 \mathrm{~ms} \\
\mathrm{TpHL}=0.033 \mathrm{~ms} \\
\text { Total } \mathrm{Tpd}=0.033 \mathrm{~ms}\end{array}$ \\
\hline For Carry & $\begin{array}{c}\mathrm{T} \mathrm{pLH}=0.1234 \mathrm{~ms} \\
\mathrm{~T} \mathrm{pHL}=0.067 \mathrm{~ms} \\
\text { Total } \mathrm{Tpd}=0.1904 \mathrm{~ms}\end{array}$ & $\begin{array}{c}\mathrm{TpLH}=0 \mathrm{~ms} \\
\mathrm{TpHL}=0 \mathrm{~ms} \\
\text { Total } \mathrm{Tpd}=0 \mathrm{~ms}\end{array}$ & $\begin{array}{c}\mathrm{TpLH}=0.033 \mathrm{~ms} \\
\mathrm{TpHL}=0 \mathrm{~ms} \\
\text { Total } \mathrm{Tpd}=0.033 \mathrm{~ms}\end{array}$ \\
\hline
\end{tabular}


Propagation delay occurs for many reasons such as high voltage supply, increase in output load capacitance, operating temperature. Capacitance needs time to get charged and discharged and in both times output can't get changed instantly which is the main parameter of the delay. CNTFET and FINFET show less time delay between switching on and switching off the circuit rather than MOSFET. But comparatively CNTFET performs best because of least value of quantum capacitance which leads to lesser propagation delay.[12]

\section{Conclusion}

Propagation delay determines the working speed feature of a circuit. The circuit which has less propagation delay can be considered as efficient. Nowadays the manufacturing process of CMOS is designed in nano-dimensions because of this issue. For the most efficient half adder circuit, CNTFET is the best alternative. But due to being expensive and highly controlling issue CNTFET has still some difficulties to get used widely but in nano scale regime, using CNTFET is much beneficiary.

\section{Journal Papers:}

\section{References}

[1]. Shaifali Ruhil 1, Komal Rohilla 2 Jyoti Sehgal 3" CNTFET Based Energy Efficient Full Adder" International Journal of Innovat ive Research in Science, Engineering and Technology (An ISO 3297: 2007 Certified Organization) Vol. 4, Issue 6, June 2015.

[2]. Deepak Kumar, ajay Kumar Dadoria, T.K. Gupta," Carbon NanoTube based logic gates structure for low power consumption at nano-scaled era" Cloud System and Big Data Engineering (Confluence), 2016 6th International Conference, 14-15 Jan. 2016.

[3]. Mingyu Zhang; John T. W. Yeow, "Nanotechnology-Based Terahertz Biological Sensing: A review of its current state and things to come”, IEEE Nanotechnology Magazine,Year: 2016, Volume: 10, Issue: 3.

[4]. Trevor C. Smith; Sergey Edward Lyshevski, " Nanotechnology for portable energy systems: Modular photovoltaics, energy storage and electronics”, 2016 IEEE 36th International Conference on Electronics and Nanotechnology(ELNANO),Year: 2016.

[5]. James E. Morris,"Nanotechnology laboratory and nanoelectronics simulation courses", 2015 IEEE Nanotechnology Materials and Devices Conference (NMDC), Year: 2015.

[6]. Richard Goering," BSIM-CMG FinFET Model - How Academia and Industry Empowered the Next Transistor",Industry Insights Blogs, 21 Jan 2013.

[7]. Juan P. Duarte; Sourabh Khandelwal; Aditya Medury; Chenming Hu; Pragya Kushwaha; Harshit Agarwal; Avirup Dasgupta; Yogesh S. Chauhan," BSIMCMG: standard FinFET compact model for advanced circuit design”, European Solid-State Circuits Conference (ESSCIRC), ESSCIRC 2015 - 41st, Year: 2015.

[8]. Yu Yuan; Cecilia Garcia Martin; Erdal Oruklu," Standard cell library characterization for FinFET transistors using BSIM-CMG models", 2015 IEEE International Conference on Electro/Information Technology (EIT) Year: 2015.

[9]. Alessandra Leonhardt; Luiz Fernando Ferreira; Sergio Bampi," Nanoscale FinFET global parameter extraction for BSIM-CMG model" Circuits \& Systems (LASCAS), 2015 IEEE 6th Latin American Symposium on, Year: 2015.

[10]. Raghav Gupta; Ashwani K. Rana," Comparative study of digital inverter for CNTFET \& CMOS technologies"2013 Nirma University International Conference on Engineering (NUiCONE), Year: 2013.

[11]. T. Ravi; V. Kannan," Modeling and performance analysis of ballistic carbon nanotube field effect transistor (CNTFET) "Recent Advances in Space Technology Services and Climate Change (RSTSCC), 2010,Year: 2010.

[12]. Raghav Gupta; Ashwani K. Rana," Study of CNTFET based basic current mirror in comparison with NMOS technologies", Advanced Computing and Communication Systems (ICACCS), 2013 International Conference on, Year: 2013. 